Check for updates

Cite this: RSC Adv., 2019, 9, 12854

\title{
Corrosion resistance for superwetting immiscible oil/water separation porous materials $\dagger$
}

\author{
Wanting Rong, (D) a Haifeng Zhang, (D) *abc Yanjing Tuo, ${ }^{\mathrm{b}}$ Weiping Chen ${ }^{\mathrm{ac}}$ \\ and Xiaowei Liuabc
}

In this paper, we first fabricate a 3D porous FZCF (FAS-modified ZnO-grown copper foam) with robust superhydrophobicity in air and superoleophilicity under water and the repeatable superwettability, and then mainly explore and analyze its corrosion resistance. The superhydrophobic-superoleophilic FZCF as an immiscible oil/organic solvent separation material shows high adsorption capacity and separation efficiency due to its heterogeneous micro-nano structures and low surface energy. It has excellent corrosion resistance under various $\mathrm{pH}$ conditions, and can serve as a corrosion protective barrier that prevents metal from contacting corrosive seawater in marine applications. Adsorbed oils also make superoleophilic FZCF keep its durability and stability after suffering attack in strong acid and alkali environments for a long time. Superwetting porous FZCF material that possesses outstanding excellent corrosion resistance demonstrates potential applications in many industrial fields such as oily wastewater treatment and marine oil spill accidents.

Received 4th March 2019

Accepted 10th April 2019

DOI: 10.1039/c9ra01632e

rsc.li/rsc-advances

sponges, ${ }^{9-11}$ membranes, ${ }^{12-15}$ foams, ${ }^{16,17}$ textiles, ${ }^{18,19}$ aerogels, ${ }^{20,21}$

\section{Introduction}

Nowadays, due to the increasing demand of environmental protection and economic development, large quantities of oily wastewater and sanitary sewage have caused serious environmental problems. In addition, oil spill accidents all over the world have resulted in the waste of precious resources and damage to the environmental system such as the Deepwater Horizon and the Gulf of Mexico oil spills, which have polluted the oceans and rivers by discharging millions of tons of crude oil..$^{1-3}$ It is extremely important to make the purity of the fuel oil reach a certain standard in the ocean transportation field, because oil mixed with a little water will increase the risk of oil exploration and finally result in serious accidents. Therefore, there exists an urgent need for effectively separating oil/water mixtures. Jiang and co-workers proposed that the superwetting polytetrafluoroethylene coated mesh could efficiently achieve oil/water separation. ${ }^{4}$ In 2017, Faze Chen etc reported a facile organic solvent free method was described to prepare a porous PVDF-MWCNT foam to separate immiscible oils/ organic solvents and aqueous solutions. ${ }^{5}$ Therefore, many superwettability materials such as coating meshes, ${ }^{6-8}$

${ }^{a}$ MEMS Center, Harbin Institute of Technology, Harbin 150001, China. E-mail: zhanghf@hit.edu.cn

${ }^{b}$ State Key Laboratory of Urban Water Resource \& Environment, Harbin Institute of Technology, Harbin 150001, China

'Key Laboratory of Micro-Systems and Micro-Structures Manufacturing, Ministry of Education, Harbin, 150001, China

$\dagger$ Electronic supplementary information (ESI) available. See DOI: 10.1039/c9ra01632e particles, ${ }^{22,23}$ and others ${ }^{24-26}$ are applied for oils/water separation. ${ }^{27-30}$ Superwetting porous copper foam with superhydrophobicity and superoleophilicity are of greatest concern since these materials are affordable and well-prepared 3 dimensional (3D) network composite structures and large surface area compared with their counterparts in the form of membrane, sponges, textiles, fiber and particle. ${ }^{31-37}$ Moreover, the material could achieve the rapid separation of sundry immiscible oil/water mixtures and high adsorption capacity with superwettability caused by the hierarchical micro-nano structures and chemical compositions, revealing the coactions of structure and performance. ${ }^{38}$ In the current study, the researches are limited to preparation and application of oil/ water separation materials. However, these materials cannot be applied in some solutions such as seawater are highly corrosive. Little attention is paid to corrosion resistance of oil/ water separation materials resulting in impractical application.

In this paper, we prepare superwetting (superhydrophobic and superoleophilic) 3D porous FZCF (FAS-modified ZnO-grown copper foam) through hydrothermal process under appropriate $\mathrm{pH}$ conditions and FAS-modification, and focus on the study of its corrosion resistance. Due to the surface giving rise to heterogeneous micro-nano structures of flower-like protrusions and petals and low surface energy, the roughened FZCF shows the repeatable superwettability that includes superhydrophobicity in air and superhydrophilicity under water. The corrosion resistance of the FZCF is tested by a potentiodynamic polarization method. The superhydrophobic FZCF has excellent corrosion resistance and superoleophilicity can largely improve 
the corrosion resistance of the copper foam in corrosive solutions. Moreover, the durability and stability of the FZCF in the practical adsorption and separation for immiscible oils/organic solvents and water also can be kept in highly acidic, alkaline, and salty environments. The FZCF as a kind of oil/water separation material has potential practical applications due to the improved corrosion resistance.

\section{Experimental section}

\subsection{Materials and reagents}

The materials used in the experimental process include Zinc acetate $\left(\mathrm{Zn}\left(\mathrm{CH}_{3} \mathrm{COO}\right)_{2} \cdot 2 \mathrm{H}_{2} \mathrm{O}\right)$, zinc nitrate $\left(\mathrm{Zn}\left(\mathrm{NO}_{3}\right)_{2} \cdot 6 \mathrm{H}_{2} \mathrm{O}\right)$, hexamethylenetetramine $\left(\mathrm{C}_{6} \mathrm{H}_{12} \mathrm{~N}_{4}\right)$, polyetherimide (PEI), fluorinated silane (FAS, $1 \mathrm{H}, 1 \mathrm{H}, 2 \mathrm{H}, 2 \mathrm{H}$-perfluorooctadecyltrichlorosilane), methylene blue and Sudan III were purchased from Aladdin Industrial Corporation (City of Industry, USA). Tetrachloromethane, toluene, hexane, chloroform, octane and tetrachloroethane used in the separation test are all of analytical grade. All the chemical reagents were analytical grade, including acetone, ammonia, ethanol, hydrochloric acid $(\mathrm{HCl})$ and so forth. Copper foam of $1 \mathrm{~mm}$ thickness was obtained from Taili foam metal Co., Ltd., Suzhou, China. Deionized water is used for washing in the experiment.

\subsection{Preparation of immiscible oil/organic solvents adsorption/separation experiments of the porous FZCF}

The preparation process of 3D porous FZCF surface could be divided into two steps that the first step was the preparation of the seed layer of $\mathrm{ZnO}$ and the second step was the growth of $\mathrm{ZnO}$ nanoflowers based on the seed layer in hydrothermal condition. Firstly, the copper foam substrate treated with hydrochloric acid and ethanol was immersed into a $5 \mathrm{mM}$ zinc acetate ethanol solution and heated on the electric heating plate for $20 \mathrm{~min}$ at $350{ }^{\circ} \mathrm{C}$ in air. The $\mathrm{ZnO}$ seed layer was generated. Secondly, a $150 \mathrm{~mL}$ mixed solution containing $100 \mathrm{mM}$ $\mathrm{Zn}\left(\mathrm{NO}_{3}\right)_{2}, 50 \mathrm{mM} \mathrm{C} \mathrm{H}_{12} \mathrm{~N}_{4}$, and a PEI solution $(0.7 \mathrm{~g})$ was prepared in a sealed high pressure reactor. The $\mathrm{pH}$ of the above mixture was adjusted to 10 with ammonium hydroxide. The assynthesized sample was inclined at $45^{\circ}$ in the mixed solution and reacted for $10 \mathrm{~h}$ at $95{ }^{\circ} \mathrm{C}$ in a thermal bath. The substrates were taken out from the reactor when the $\mathrm{ZnO}$ nanoflowers deposition was complete and substrates were washed with deionized water and dried at room temperature. Finally, the sample was immersed in an ethanol solution of $0.5 \mathrm{wt} \%$ FAS for $60 \mathrm{~min}$ and dried in a drying oven at $120{ }^{\circ} \mathrm{C}$ for $60 \mathrm{~min}$.

Acids or alkalis were added in deionized water to adjust different $\mathrm{pH}$ values, and then the superwetting behavior and stability of the porous FZCF were investigated in as-prepared solutions. The adsorption/separation experimental equipment has been set up to study corrosion resistance of the FZCF in mixing immiscible oils/organic solvents.

In order to investigate the adsorption capacity of the as prepared FZCF toward oil/organic solvent and water mixtures, selective adsorptions experiments are carried out using both light and heavy oil/organic solvents. Two organic solvents with different density, chloroform (heavy organic solvent) and toluene (light organic solvent), are chosen as model adsorbates to demonstrate the adsorption capacity of the porous FZCF toward different oils/organic solvents in Fig. S4. $\dagger$ Movie S1 (ESI $\dagger$ ) shows eleven examples of light/heavy oils and organic solvents rapid removal from water surface and underwater, in which Sudan III dyed all the oil/organic solvents are set as the exemplar oil drops and dispersed on water surface and underwater (the water is dyed with methylene blue). The other nine adsorption experiments (Movie S1, ESI $\dagger$ ) also have the same effect in capturing oils/organic solvents from water with high adsorption capacity.

To test adsorption capacity oil/organic solvents $(M)$, the porous FZCF foam is immersed in various oil/organic solvents at room temperature and then fast taken out for weight measurements after complete adsorption. The adsorption capacity $(M)$ is evaluated according to the following eqn (1):

$$
M=\left(\frac{m_{1}}{m_{0}}-1\right) \times 100 \%
$$

where $m_{0}$ and $m_{1}$ are the weight of the FZCF before and after adsorption respectively. At least five measurements are processed for oil/organic solvent to gain the average value.

The separation experiment (Fig. 4a) is performed to validate the gravity driven oils/organic solvents and water separation capability of FZCF and also evaluate the oil/water separation efficiency (SE\%) of FZCF. The labelled oils (15 mL) and water (15 $\mathrm{mL}$ ) placed in a beaker form a layered oil/water mixture. In order to distinguish water from oils (toluene), the water is dyed with methylene blue. The sample is washed with ethanol, and then dried in vacuum oven after each separation.

When the toluene-water mixture is poured onto the FZCF sample, toluene quickly permeated through the FZCF sample and rapidly dropped into the conical flask and the water dyed blue is blocked below (Movie S2, ESI $\dagger$ ). The separated toluene and water are collected from the conical flask and the glass funnel, respectively. Meanwhile, there is no visible water (dyed with methylene blue) in the toluene and retaining above the FZCF sample due to its superhydrophobicity and superoleophilicity, indicating a very high and stable separation efficiency.

The separation efficiency (SE\%) of FZCF is calculated according to eqn (2) as follows

$$
\mathrm{SE} \%=\left(\frac{m_{\mathrm{b}}}{m_{\mathrm{a}}}\right) \times 100 \%
$$

where $m_{\mathrm{a}}$ is the mass of water in the toluene-water mixture before the separation and $m_{\mathrm{b}}$ is the mass of water in the glass funnel after the separation.

The chemical durability of the FZCF is further demonstrated by measuring the oil adsorption in highly acidic, alkaline, and salty environments. Concentrated $\mathrm{HCl}(100 \mathrm{~mL}), \mathrm{NaOH}(100$ $\mathrm{mL}$ ), and saturated ( $\approx 26.5 \%$ by weight) $\mathrm{NaCl}$ solution $(100 \mathrm{~mL})$ are prepared as the corrosive aqueous solutions simulating contaminated seawater here.

The corrosion resistance of the FZCF was investigated by a potentiodynamic polarization technology. The polarization 
data were recorded in $3.5 \mathrm{wt} \% \mathrm{NaCl}$ solution after 7 days immersion.

\section{Results and discussion}

3.1 Surface morphology, chemical characterization and superwettability in different $\mathrm{pH}$ solutions of the porous FZCF

In order to investigate whether the reaction process provided enough surface roughness and furthermore produced enough superwettability, the surface morphology of the prepared FZCF is characterized by scanning electron microscopy (SEM). The surface morphologies were observed using a field-emission scanning electron microscope (FE-SEM, TESCAN VEGA). Fig. 1d shows a low-resolution scanning electron microscopy (SEM) image of the as-prepared FZCF with a pore size of $300 \mu \mathrm{m}$ and the porosity of $98 \%$. Fig. 1e shows tiny heterogeneous protrusions often form dense growths on the porous FZCF skeleton. At higher resolution, the porous FZCF surface is uniformly covered by hierarchical micro-nano structures of flower-like protrusions and petals which are presumably caused by the spherical structures of $\mathrm{ZnO}$ (Fig. 1f). The local magnification image (Fig. 1g-h) further indicates the flower-like structures of the FZCF similar with the physical photos of the petals of Sedeveria silver frost (Fig. 1a-b). The petals of the flower-like structures are almost 200-300 nm in diameter (Fig. 1g) with its multilayered structure consisting of hierarchically packed nanoslices and the thickness of nanoslices is estimated to be $80 \mathrm{~nm}$ (Fig. 1h). Due to Sedeveria silver frost has the special wettabilities that the leaves are hydrophobic, such a biomimetic hierarchical micro-nano structures of flower-like protrusions and petals can help supporting water droplets (Fig. 1c) on the FZCF and is vitally essential for the superhydrophobicity of the FZCF.
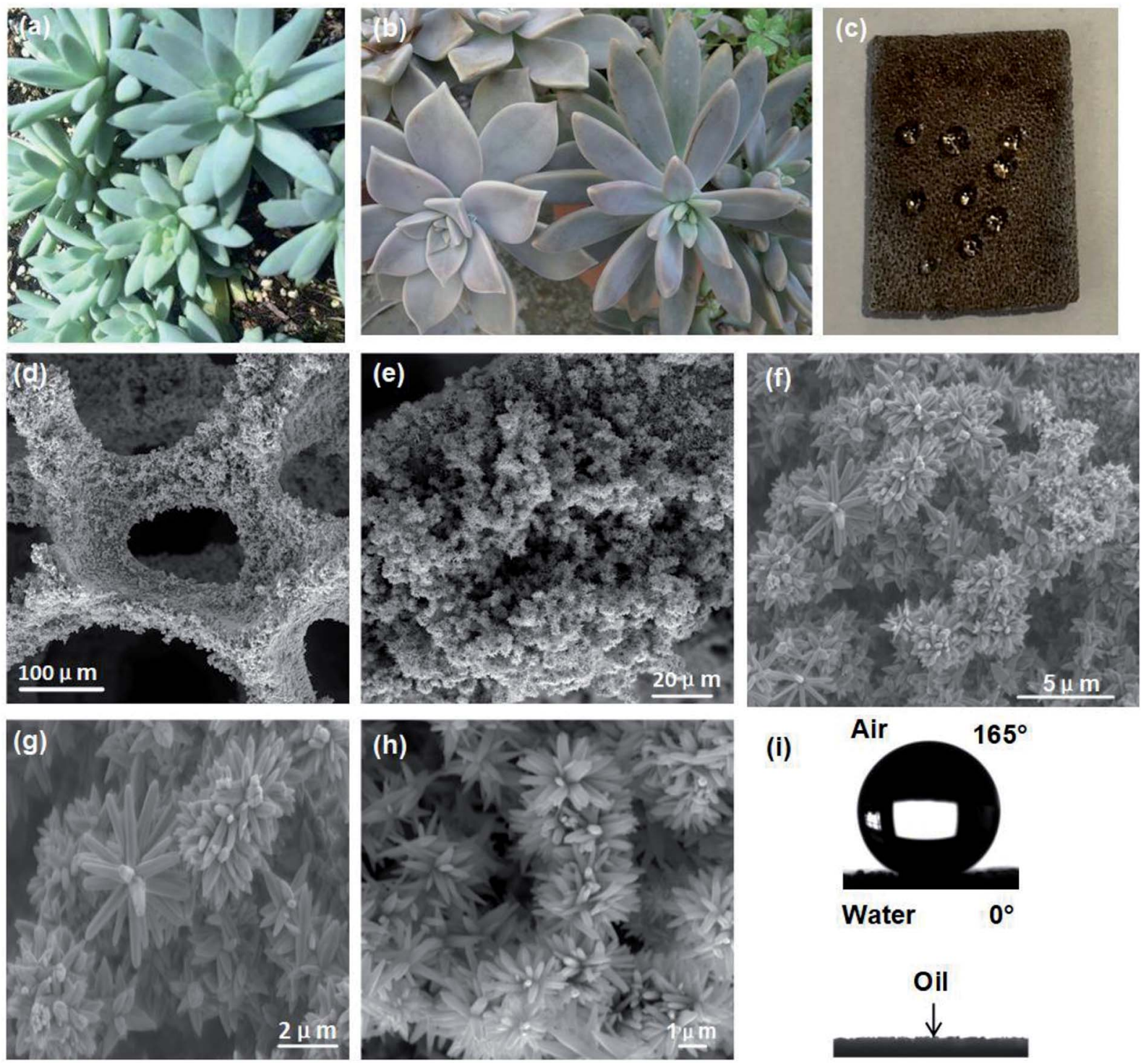

(i)
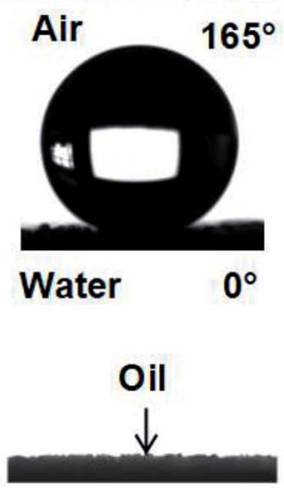

Fig. 1 (a) and (b) Photos of the petals of Sedeveria silver frost. (c) Picture of as-prepared porous FZCF with water droplets on top. (d-h) SEM images with different magnifications of the porous FZCF sample (10 $\mu \mathrm{m}, 2 \mu \mathrm{m}$ and $1 \mu \mathrm{m}, 500 \mathrm{~nm}$ and $200 \mathrm{~nm})$. (i) Optical images of water droplets deposited on corresponding surface showing the superhydrophobicity in air and superhydrophilicity under water. 
The CA and SA of water droplets were measured by an optical contact-angle meter system (Data Physics Instrument $\mathrm{GmbH}$, Germany) at ambient temperature. The water CA and SA reported were the mean values measured with $5 \mu \mathrm{L}$ of water droplets at five different positions on each test surface. The average CA value was an average of the five measurements at different spots of the same sample. The corresponding water contact angle (CA) measurements on the sample surface show that the porous FZCF is both superhydrophobic in air and superoleophilic in water in Fig. 1i. FZCF has a static water CA in air of $165^{\circ}$ and the water droplet quickly permeates with a static oil CA in water of $0^{\circ}$ indicating superhydrophobicity and superoleophilicity.

EDS of the sample surface is also detected in a random area eight times (Fig. 2a). The corresponding element distributions were determined by energy dispersive X-ray spectroscopy (EDX). The elemental analysis performed using energy dispersive X-ray spectroscopy (EDS; attached to SEM) on as-prepared FZCF sample shows the major element content included zinc, copper, carbon oxygen, fluorine and silicon based on quantitative analysis. The results are incapable of being perceived as different indicating that the $\mathrm{ZnO}$ flower-like heterogeneous structures are uniformly distributed on the FZCF surface.

The X-ray diffraction (XRD) patterns of the sample were performed on an Empyrean diffractometer (Panalytical, Dutch) using $\mathrm{Cu} \mathrm{K} \alpha_{1}$ radiation $(\lambda=0.15418 \mathrm{~nm})$ with the $2 \theta$ ranging from $20^{\circ}$ to $80^{\circ}$. The X-ray powder diffraction (XRD) is utilized to characterize the composition of micro-nano structures formed over the FZCF sample. XRD pattern of the FZCF sample are shown in Fig. 2b. Diffraction peaks of the sample are all indexed to pure copper [PDF file: 04-0836] and zinc oxide [PDF file: 361451], and no other crystal phases exist. According to the XRD patterns of the copper foam after hydrothermal procedure, the three strong peaks located at $31.769^{\circ}, 34.421^{\circ}$ and $36.252^{\circ}$ are

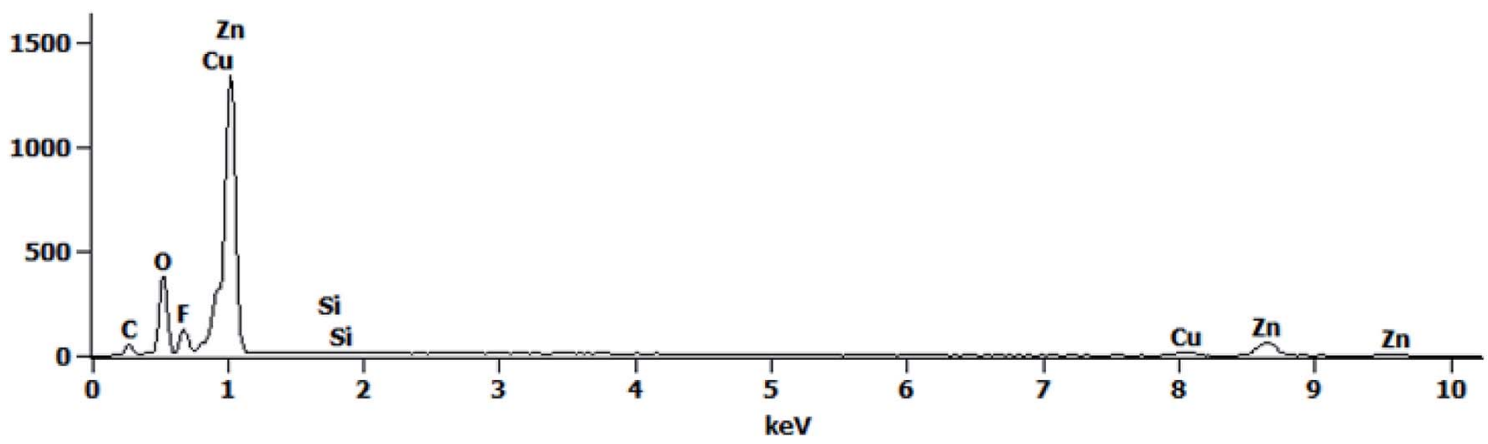

(a)

(b)

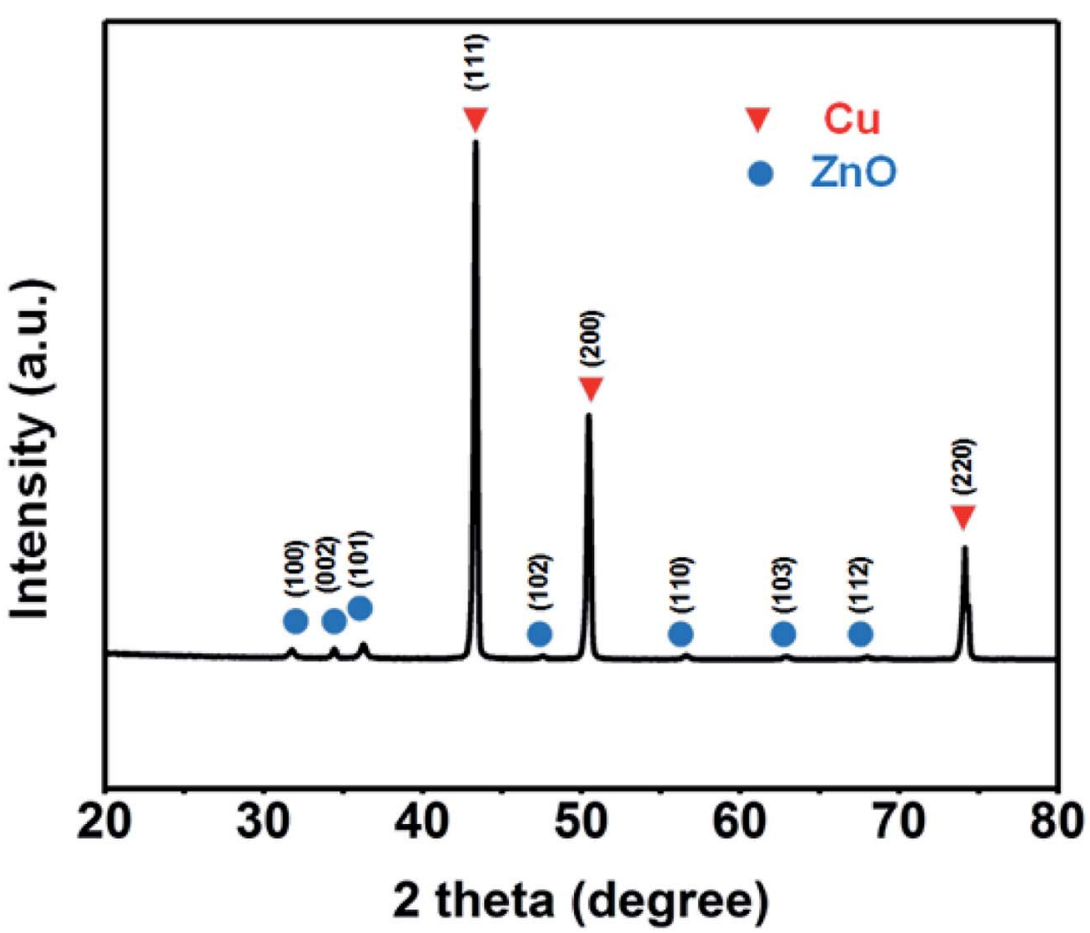

Fig. 2 (a) The EDS spectrum from an as-prepared sample. (b) XRD patterns of the FZCF. All the peaks can be indexed to hexagonal phase of zinc and pure copper. 
ascribed to (100), (002) and (101) planes of $\mathrm{ZnO}$, respectively. The peaks ascribed to (102), (110), (103) and (112) planes of $\mathrm{ZnO}$ can be observed from the XRD patterns. It is indicated that $\mathrm{ZnO}$ is the main composition of micro-nano structures formed on the copper foam substrate after hydrothermal procedure. After the FZCF preparation, three strong peaks of $\mathrm{Cu}$ ascribed to (111), (200) and (220) planes are also observed in Fig. 3. The result reveals that the main compositions of copper foam substrate remain unchanged. Namely, the hierarchical flowerlike structures of $\mathrm{ZnO}$ grown on the surfaces of the asprepared FZCF are at the micro-nano scale, and the bulk of the sample is $\mathrm{Cu}$.

Other chemical characterization results of the prepared FZCF also reveal the mechanism of the improved superwetting performances by means of XPS, FTIR and Roman spectral analysis (Fig. S1-S3, ESI $\dagger$ ) for providing the basis to perfecting the immiscible oils/organic solvents separation ability and the adsorption capacity (or chemical durability and stability).

The surface morphology has influenced on wettability that can be explained by Cassie-Baxter equation. The equation demonstrates that the air trapped in the rough surface will greatly improve the wettability. ${ }^{39}$ Due to the porosity of FZCF, it is in a combined Cassie-Wenzel state on the surface that the water droplets penetrate into the pores in some extent leaving air pockets below it. Herein, estimating the solid fraction of solid/water interface per unit area is 0.15 (based on SEM image shown in Fig. 1) demonstrating that more than $89 \%$ of the contact area belongs to the air/solid interface. Due to the solid fraction of solid/water interface is smaller than that of air/solid interface, the tiny heterogeneous protrusions grown on the porous FZCF skeleton, air and liquid phase can increase the bulk of the air layer to improve the superhydrophobicity in a combined Cassie-Wenzel state shown in Fig. 3a. Based on three phase contact line, the FZCF shows favorable superhydrophobicity in air and can be utilized in immiscible oil/organic solvents and water adsorption and separation.

Moreover, the stability in acidic and alkaline environments of the practical separation for immiscible oils/organic solvents and water is investigated as well. As-prepared FZCF with different $\mathrm{pH}$ values solutions droplets on top is shown in Fig. 3 b. It can keep the static contact angles which are more than $150^{\circ}$. When the as-prepared FZCF samples are immersed into in deionized water with different $\mathrm{pH}$ values (1 to 13) for 24 hours, the FZCF sample are still superhydrophobic with static WCAs higher than $152^{\circ}$, indicating the FZCF is insusceptible to the extreme acid and alkaline environments (Fig. 3c). For some strong acid or alkali solutions ( $\mathrm{pH}$ value is $1,3,11$ and 13), the CAs decline to less than $153^{\circ}$, demonstrating that the chemical damage has influenced the superhydrophobicity of the FZCF surfaces in harsh circumstances. When the $\mathrm{pH}$ value ranges from 4 to 6 , the superhydrophobicity of the FZCF is improved and the contact angles are able to reach $160^{\circ}$ in comparative faintly acid ( $\mathrm{pH}$ value is 5), neutral solutions ( $\mathrm{pH}$ value is 7 ), and alkalescence solutions ( $\mathrm{pH}$ value is 9). From a view of practical application, these results reveal that the superwetting FZCF has the chemical durability and stability for oil/organic solvents and water separation, further proving that can utilize for industrial oily wastewater treatment and marine oil spill accidents.

\subsection{Immiscible oils/organic solvents and water adsorption/ separation experiments of the porous FZCF}

The superoleophilicity and superhydrophobicity make the porous FZCF exhibits excellent adsorption capacities towards various oils and organic solvents, ranging from 17.0 to 31.0 times of its own weight based on the tested organic liquids (Fig. S5, ESI $\dagger$ ). The rapid oil/water adsorption behavior of the porous FZCF either on water or underwater can be attributed to (a)

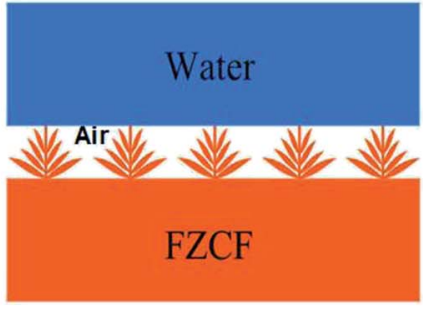

(b)

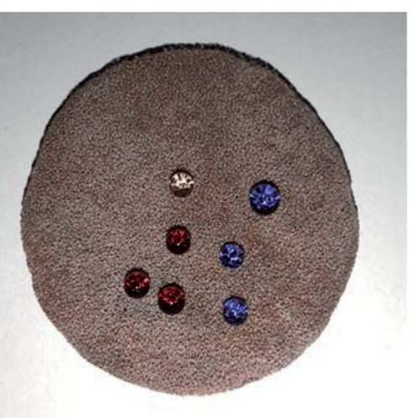

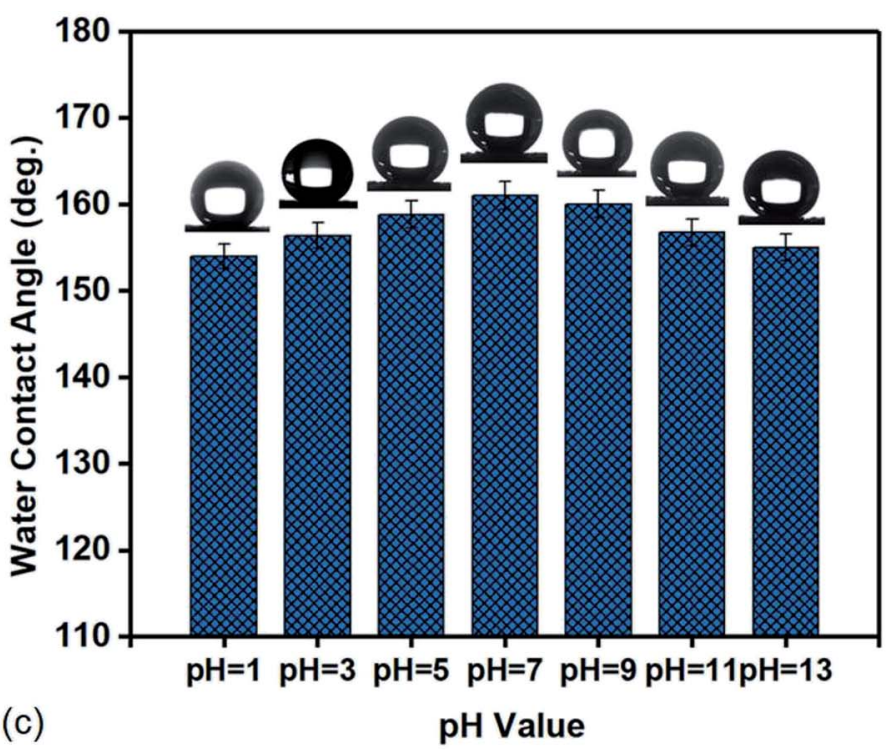

Fig. 3 (a) Schematic of the superhydrophobic porous FZCF sample. (b) Picture of as-prepared FZCF with different pH values solutions droplets on top. (c) Static water contact angles of the FZCF in different $\mathrm{pH}$ values solutions. 
its the porous micro-nano structures of flower-like protrusions and superhydrophobic/superoleophilic properties. ${ }^{40} \mathrm{Oil} /$ organic solvents are adsorbed in the pores formed by the layered foam skeleton of the FZCF while water is completely repelled by the superhydrophobic surface. Due to the capillarity and low surface energy, the superoleophilicity and superhydrophobicity is further reinforced when the oil molecules spread into the inner pores of the FZCF modified with the FAS on the layered foam skeleton.

Fig. 4b shows the toluene-water separation efficiency and the water contact angles (WCAs) of the FZCF sample after 40 recycling separation times. Even though the separation efficiency and the water contact angles gradually decrease (which can be attributed to abrasion of the superhydrophobic FZCF sample surface during the separation process) when the FZCF sample is applied to separate toluene and water. It still holds remarkable superhydrophobic properties $\left(>152^{\circ}\right)$ after 40 separation cycles, meanwhile the separation efficiency remains at above $96.1 \%$. More importantly, FZCF can be retrieved after the absorbed oil is easily removed by washing with absolute alcohol and then dried in vacuum oven after each separation, demonstrating the outstanding recyclability of adsorption/ desorption and anti-fouling property of the FZCF material. Fig. $4 \mathrm{c}$ and d shows the SEM images of the FZCF sample after 5 and 30 recycle times separation tests. Though the FZCF surface is contaminated by oils/organic solvents and the FAS modified on the FZCF is destroyed in a certain extent, the superhydrophobic-superoleophilic property of the FZCF just decrease a little resulting in keeping oil-water separation performance after multiple separation times. By contrast, the
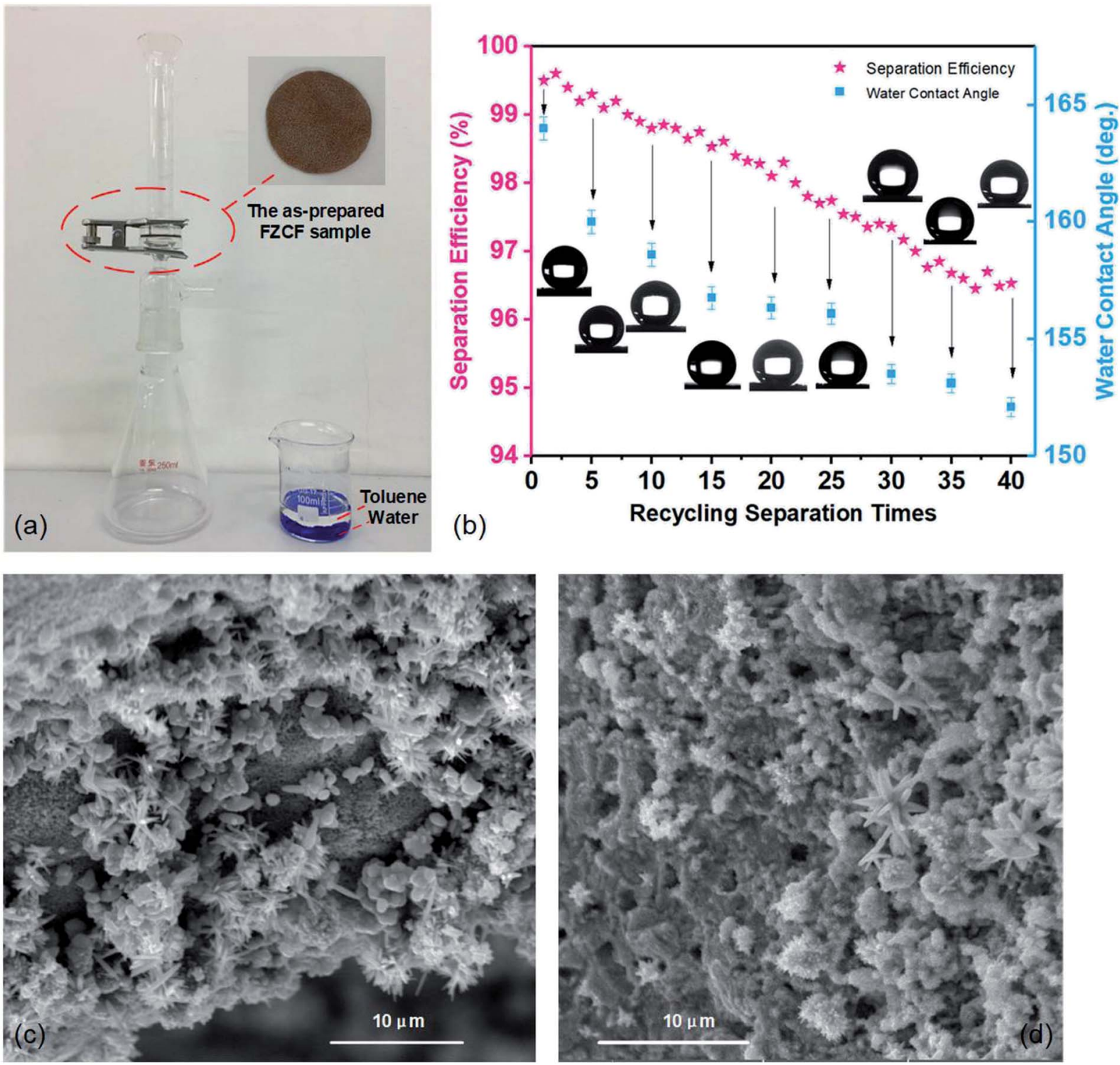

Fig. 4 (a) The solely gravity driven oil/water separation test of the FZCF. (b) The oil/water separation efficiency and water contact angle as a function of 40 recycle times by taking the toluene-water mixture as an example. (c) and (d) SEM images the FZCF sample after 5 and 30 recycle times separation tests. 
surface morphology has no huge changes such as micro-nano structures, and it still proves the chemical durability of the porous FZCF.

Fig. 5 shows the adsorption capacity and separation efficiency of FZCF for three typical organic liquids (motor oil, hexane and toluene) in different $\mathrm{pH}$ solutions. Motor oil, hexane, and octane are added into the corrosive aqueous solutions respectively. Then the oil/organic liquid droplets are removed by the FZCF sample rapidly. The results indicate that the adsorption capacity was still above 20 for motor oil in strong acid and strong alkaline. Others keep on a steady value of 20 and 25, respectively. As shown in Fig. 5b, the motor oil has the highest separation efficiency of $99.2 \%$ under neutral conditions compared with the other two corresponding to Fig. 5a. SE\% of three oils are all up to above $96.4 \%$ in strong acid and strong alkaline. The as-prepared FZCF shows robust separation performance for various light oils and heavy oils (Fig. S5, ESI $\dagger$ ). For these oils, the separation efficiencies range from the $97.8 \%$ to $99.4 \%$. The result suggests that the as-prepared FZCF can easily remove oil/organic solvents either on water or underwater or separate both. The chemical durability of the FZCF can be verified also demonstrates the feasibility of the proposed method for preparing advanced oil/water separation FZCF material.

\subsection{Corrosion resistance analysis of the porous FZCF in harsh circumstances}

The polarization curves (Tafel) presented in Fig. 6a show that the untreated surface, cleaned FZCF and adsorbed-oils FZCF reveals a narrow passive range and a breakdown between $-0.2 \mathrm{~V}$ and $-0.3 \mathrm{~V}$, where the current density increases abruptly with slowly increased anodic polarization potential.

The corrosion potential $\left(E_{\text {corr }}\right)$ of the untreated surface is $-0.305 \mathrm{~V}$ and its corrosion current density $\left(I_{\text {corr }}\right)$ is $8.5 \times 10^{-7} \mathrm{~A}$ $\mathrm{cm}^{-2}$. While, after the formation of superhydrophobic FZCF, the $E_{\text {corr }}$ changes to $-0.232 \mathrm{~V}$ and the $I_{\text {corr }}$ of cleaned FZCF decreases slightly to $4.82 \times 10^{-9} \mathrm{~A} \mathrm{~cm}^{-2}$ due to the superhydrophobic $\mathrm{ZnO}$ layer reduces the corrosion rate and protects the underneath copper foam from corrosion attack, which indicated that the improvement of the protective properties of the superhydrophobic FZCF generated significant alterations in the corrosion behaviour.

If the cleaned FZCF had adsorbed immiscible oils/organic solvents, the $E_{\text {corr }}$ shifts positively to $-0.215 \mathrm{~V}$. Simultaneously, the $I_{\text {corr }}$ of the adsorbed-oils FZCF decreases to $3.18 \times$ $10^{-9} \mathrm{~A} \mathrm{~cm}^{-2}$ which is less than that of the untreated surface and cleaned FZCF. Therefore, the adsorbed-oils FZCF further enhances the corrosion protection by adsorbing oils as a protective layer. The corrosion potential moving to right demonstrates that both trapped air and adsorbed oils are good physical barriers to isolate copper foam from $3.5 \% \mathrm{NaCl}$ aqueous solution in a short time. It can be concluded that cleaned FZCF and adsorbed-oils FZCF have the potential to inhibit the corrosion of the copper foam substrates. The adsorbed-oils FZCF is more effective than the air layer to protect the copper foam from corrosion. The protective capability of the FZCF probably originates from both the superhydrophobicity and superoleophilicity based the physical diffusion barriers.

To further explore the corrosion inhibition capability of the superhydrophobic FACF, the corrosion protection performance of the FZCF was also studied by the electrochemical impedance spectra (EIS). Fig. $6 \mathrm{~b}$ displays the Bode-modulus plots untreated copper foam, cleaned FZCF and adsorbed-oils FZCF. The $|Z|$ decreases with the decrease of frequency $(\mathrm{Hz})$ in solution for 7 days, indicating the responses of inductive impedance at low frequencies. The $|Z|$ value of the adsorbed-oils FZCF is $c a .1$ and ca. 2 orders of magnitude larger than the untreated copper foam, cleaned FZCF in the low frequency region and high frequency region. The result reflects that adsorbed oils endows FZCF an excellent anti-corrosion property, which is consistent well with the result from Tafel curves.

The Bode-phase plots presented in Fig. 6c show a welldefined time-constant at high frequencies $(\mathrm{Hz})$ throughout the whole immersion period. High frequency capacitive loop is ascribed to charge transfer of the corrosion process, while the inductive loop at low frequencies is attributed to stability layer by adsorbed intermediate products of the corrosion reaction on the electrode surface. ${ }^{41}$ For superhydrophobic cleaned FZCF,
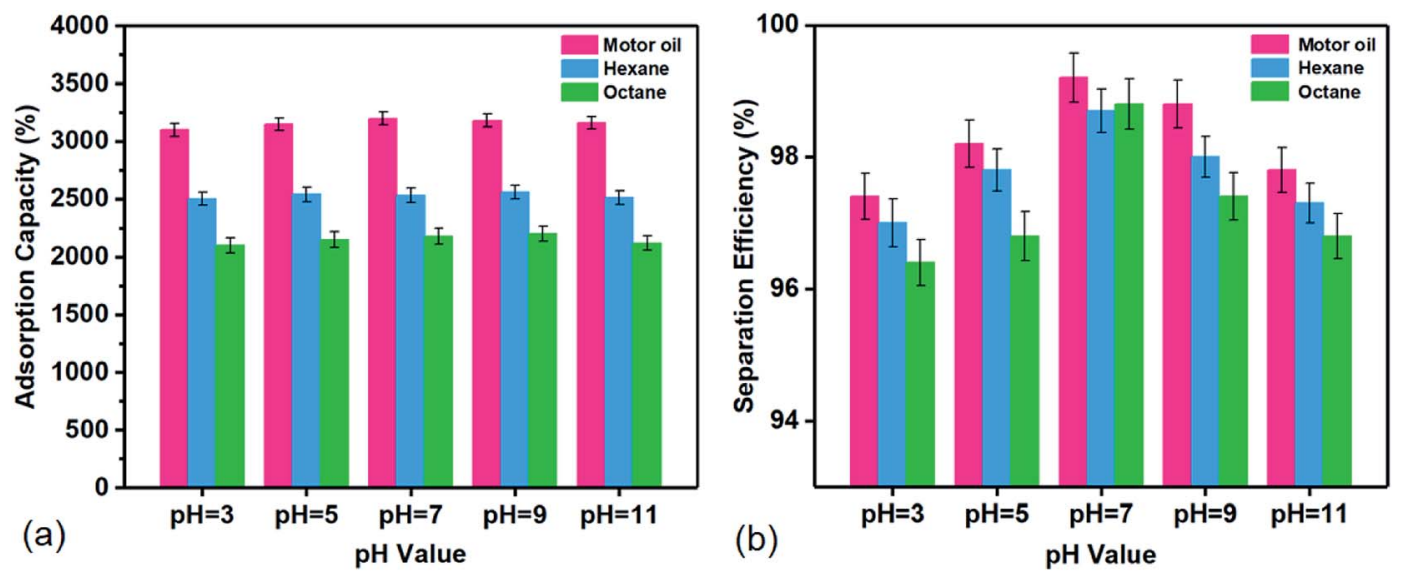

Fig. 5 (a) Adsorption capacity and (b) separation efficiency of the FZCF for different oil/organic solvents in different pH value aqueous solutions. 

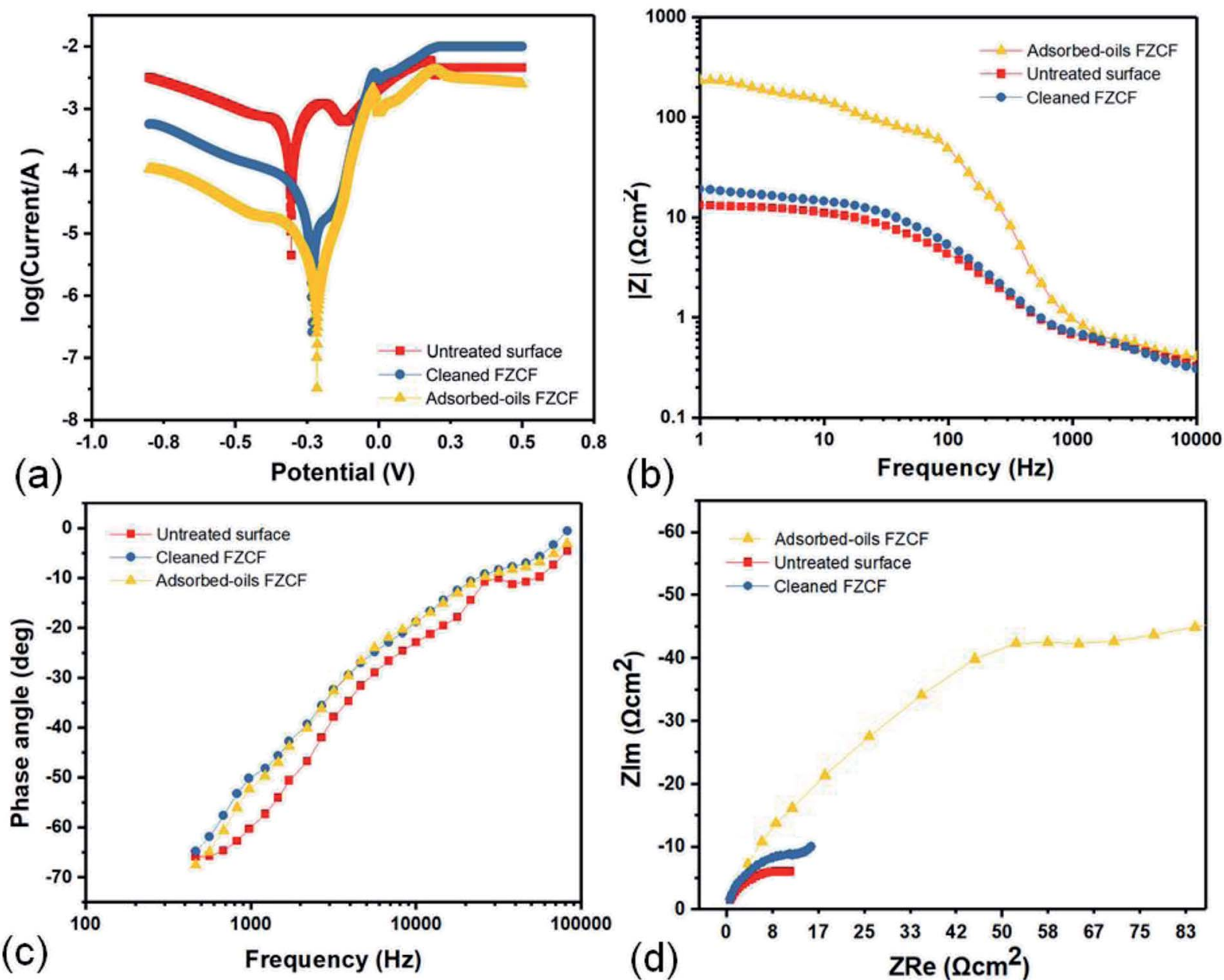

Fig. 6 (a) Tafel polarization curves (b) Bode-modulus plots and (c) Bode-phase plots of the untreated surface, cleaned FZCF and adsorbed-oils FZCF, after 7 days of immersion in 3.5 wt\% NaCl solution. (d) Nyquist plots of the untreated surface cleaned FZCF and adsorbed-oils FZCF immersion in simulated seawater for 7 days.

only a capacitive character is observed in the Bode-phase plots. As the capacitive responses appear at high frequencies, they can be attributed to the responses of protective superhydrophobic cleaned FZCF. After 7 days water immersion, Cassie-to-Wenzel wetting transition would occur to cleaned FZCF. The EIS spectra reveals that superhydrophobic cleaned FZCF can serve as a good corrosion protection coating. Similarly, it is inferred that adsorbed-oils FZCF still has a high corrosion resistance though the wetting transition from Cassie wetting state to Wenzel wetting state compared to the superhydrophobic FZCF.

The Nyquist plots in Fig. $6 \mathrm{~d}$ show three depressed semicircles in the high frequency range during further immersion (7 days). The impedance spectra of the adsorbed-oils FZCF shows a large impedance semicircle whose diameter is around several hundreds of $\mathrm{k} \Omega \mathrm{cm}^{2}$, while the impedance semicircle diameter of the cleaned FZCF and the untreated surface is dozens of $\mathrm{k} \Omega \mathrm{cm}^{2}$. These large impedance semicircles indicate that three samples all have good anti-corrosion properties after immersion for 7 days, especially the adsorbed-oils FZCF. Therefore, the adsorbedoils FZCF presents an excellent corrosion resistance after suffering the attack in $\mathrm{NaCl}$ aqueous solution for a long time.
These results demonstrate that superhydrophobic-superoleophilic FZCF has excellent corrosion resistance and oils can largely improve the corrosion resistance of the copper foam in $\mathrm{NaCl}$ aqueous solution.

\section{Conclusions}

In summary, we prepared a 3D superwetting porous FZCF with robust superhydrophobicity in air and superoleophilicity under water for adsorption/separation of immiscible oil/water mixtures and corrosion resistance tests were carried out in different environments. The porous FZCF exhibits excellent adsorption capacity, separation efficiency and robust corrosion resistance. Based on potentiodynamic polarization tests, superhydrophobic-superoleophilic FZCF has excellent corrosion resistance. The corrosion resistance of FZCF is ascribed to the combined protection effect of the trapped air film inside the superhydrophobic FZCF in various $\mathrm{pH}$ conditions, which can serve as a corrosion protective barrier that prevents metal from contacting with corrosive seawater in marine. What's more, adsorbed-oils make superoleophilic FZCF keep this property 
after suffering the attack in $\mathrm{NaCl}$ aqueous solution for a long time compared to untreated copper foam. Furthermore, the durability and stability of the FZCF in the practical adsorption and separation for immiscible oils/organic solvents and water also can be verified in highly acidic, alkaline, and salty environments. We expect that the superwetting anti-corrosion FZCF material can be further utilized as a promising candidate for oil/ water mixtures separation, industrial oily wastewater treatment and oil removal in marine oil spill accidents and oil-repelling pipeline manufacture for industrial water transportation.

\section{Conflicts of interest}

The authors declare no conflict of interest

\section{Acknowledgements}

The work was sponsored by National Science Foundation of China (No. 61474034), National Basic Research Program of China (No. 2012CB934100), Natural Science Foundation of Heilongjiang Province of China (No. F201418), State Key Laboratory of Urban Water Resource and Environment (Harbin Institute of Technology) (No. 2016TS 06).

\section{References}

1 M. A. Shannon, P. W. Bohn, M. Elimelech, J. G. Georgiadis, B. J. Marinas and A. M. Mayers, Science and technology for water purification in the coming decades, Nature, 2008, 452, 301-310.

2 J. C. Yi, F. C. Mei, C. L. Law and D. G. Hassell, A review on anaerobic-aerobic treatment of industrial and municipal wastewater, Chem. Eng., 2009, 155, 1-18.

3 J. Yuan, X. Liu, O. Akbulut, J. Hu, S. L. Suib, J. Kong, et al. Superwetting nanowire membranes for selective absorption, Nat. Nanotechnol., 2008, 3, 332-336.

4 L. Feng, Z. Zhang, Z. Mai, Y. Ma, B. Liu, L. Jiang, et al. A Super-Hydrophobic and Super-Oleophilic Coating Mesh Film for the Separation of Oil and Water, Angew. Chem., Int. Ed., 2010, 43, 2012-2014.

5 F. Chen, Y. Lu, X. Liu, J. Song, G. He, M. K. Tiwari, et al. Table Salt as a Template to Prepare Reusable Porous PVDFMWCNT Foam for Separation of Immiscible Oils/Organic Solvents and Corrosive Aqueous Solutions, Adv. Funct. Mater., 2017, 27, 1702926.

6 Y. Dong, J. Li, L. Shi, X. Wang, Z. Guo and W. Liu, Underwater superoleophobic graphene oxide coated meshes for the separation of oil and water, Chem. Commun., 2014, 50, 5586-5589.

7 N. Liu, M. Zhang, W. Zhang, Y. Cao, Y. Chen, X. Lin, et al. Ultralight Free-Standing Reduced Graphene Oxide Membranes for Oil-in-Water Emulsions Separation, J. Mater. Chem. A, 2015, 3, 20113-20117.

8 M. Cao, J. Xiao, C. Yu, K. Li and L. Jiang, Hydrophobic/ Hydrophilic Cooperative Janus System for Enhancement of Fog Collection, Small, 2015, 11, 4379-4384.
9 L. Li, B. Li, L. Wu, X. Zhao and J. Zhang, Magnetic, superhydrophobic and durable silicone sponges and their applications in removal of organic pollutants from water, Chem. Commun., 2014, 50, 7831-7833.

10 M. Khosravi and S. Azizian, Synthesis of a Novel Highly Oleophilic and Highly Hydrophobic Sponge for Rapid Oil Spill Cleanup, ACS Appl. Mater. Interfaces, 2015, 7, 2532625333.

11 L. Wu, L. X. Li, B. C. Li, J. Zhang and A. Q. Wang, Magnetic, Durable and Superhydrophobic Polyurethane @ Fe3O4 @ SiO2 @ Fluoropolymer Sponges for Selective Oil Absorption and Oil/Water Separation, ACS Appl. Mater. Interfaces, 2015, 7, 4936-4946.

12 A. K. Kota, G. Kwon, W. Choi, J. M. Mabry and A. Tuteja, Hygro-responsive membranes for effective oil-water separation, Nat. Commun., 2012, 3, 1025.

13 W. Zhang, Z. Shi, F. Zhang, X. Liu, J. Jin and L. Jiang, Superhydrophobic and superoleophilic PVDF membranes for effective separation of water-in-oil emulsions with high flux, Adv. Mater., 2013, 25, 2071-2076.

14 Y. Cao, Y. Chen, N. Liu, X. Lin, L. Feng and Y. Wei, Musselinspired chemistry and Stober method for highly stabilized water-in-oil emulsions separation, J. Mater. Chem. A, 2014, 2, 20439-20443.

15 S. J. Gao, Z. Shi, W. B. Zhang, F. Zhang and J. Jin, Photoinduced superwetting single-walled carbon nanotube/TiO(2) ultrathin network films for ultrafast separation of oil-in-water emulsions, ACS Nano, 2014, 8, 6344-6352.

16 X. Dong, J. Chen, Y. Ma, J. Wang, M. B. Chanpark, X. Liu, et al. Superhydrophobic and superoleophilic hybrid foam of graphene and carbon nanotube for selective removal of oils or organic solvents from the surface of water, Chem. Commun., 2012, 48, 10660.

17 N. Chen and Q. Pan, Versatile fabrication of ultralight magnetic foams and application for oil-water separation, ACS Nano, 2013, 7, 6875-6883.

18 J. Zhang, B. Li, L. Wu and A. Wang, Facile preparation of durable and robust superhydrophobic textiles by dip coating in nanocomposite solution of organosilanes, Chem. Commun., 2013, 49, 11509-11511.

19 S. Li, J. Huang, M. Ge, C. Cao, S. Deng, S. Zhang, et al. Self-Cleaning Cotton: Robust Flower-Like $\mathrm{TiO}_{2}$ (a) Cotton Fabrics with Special Wettability for Effective Self-Cleaning and Versatile Oil/Water Separation, Adv. Mater. Interfaces, 2015, 2, 1570068.

20 J. T. Korhonen, M. Kettunen, R. H. A. Ras and O. Ikkala, Hydrophobic Nanocellulose Aerogels as Floating, Sustainable, Reusable, and Recyclable Oil Absorbents, ACS Appl. Mater. Interfaces, 2011, 3, 1813.

21 Y. Si, Q. Fu, X. Wang, J. Zhu, J. Yu, G. Sun, et al. Superelastic and Superhydrophobic Nanofiber-Assembled Cellular Aerogels for Effective Separation of Oil/Water Emulsions, ACS Nano, 2015, 9, 3791.

22 L. P. Xu, X. Wu, J. Meng, J. Peng, Y. Wen, X. Zhang, et al. Papilla-like magnetic particles with hierarchical structure 
for oil removal from water, Chem. Commun., 2013, 49, 87528754 .

23 B. Wang, Y. Liu, Y. Zhang, Z. Guo, H. Zhang and J. H. Xin, Nanoparticles: Bioinspired Superhydrophobic $\mathrm{Fe}_{3} \mathrm{O}_{4} @$ Polydopamine@Ag Hybrid Nanoparticles for Liquid Marble and Oil Spill, Adv. Mater. Interfaces, 2015, 2, 1500234.

24 K. Li, J. Ju, Z. Xue, J. Ma, L. Feng, S. Gao, et al. Structured cone arrays for continuous and effective collection of micron-sized oil droplets from water, Nat. Commun., 2013, 4, 2276.

25 C. Luo and X. Heng, Separation of oil from a water/oil mixed drop using two nonparallel plates, Langmuir, 2014, 30, 10002-10010.

26 L. P. Xu, B. Dai, J. Fan, Y. Wen, X. Zhang and S. Wang, Capillary-driven spontaneous oil/water separation by superwettable twines, Nanoscale, 2015, 7, 13164-13167.

27 J. Ge, H. Zhao, H. Zhu, J. Huang, L. Shi and S. Yu, Advanced Sorbents for Oil-Spill Cleanup: Recent Advances and Future Perspectives, Adv. Mater., 2016, 28, 10459.

28 Q. Ma, H. Cheng, A. G. Fane, R. Wang and H. Zhang, Recent Development of Advanced Materials with Special Wettability for Selective Oil/Water Separation, Small, 2016, 12, 21862202.

29 B. Wang, W. Liang, Z. Guo and W. Liu, Biomimetic superlyophobic and super-lyophilic materials applied for oil/ water separation: a new strategy beyond nature, Chem. Soc. Rev., 2015, 44, 336-361.

30 Y. Yu, H. Chen, Y. Liu, V. S. J. Craig and Z. Lai, Selective separation of oil and water with mesh membranes by capillarity, Adv. Colloid Interface Sci., 2016, 235, 46-55.

31 D. D. Nguyen, N. H. Tai, S. B. Lee and W. S. Kuo, Superhydrophobic and superoleophilic properties of graphene-based sponges fabricated using a facile dip coating method, Energy Environ. Sci., 2012, 5, 7908-7912.

32 Y. Jin, P. Jiang, Q. Ke, F. Cheng, Y. Zhu and Y. Zhang, Superhydrophobic and superoleophilic polydimethylsiloxane-coated cotton for oil-water separation process: an evidence of the relationship between its loading capacity and oil absorption ability, $J$. Hazard. Mater., 2015, 300, 175-181.

33 J. Li, C. Xu, Y. Zhang, R. Wang, F. Zha and H. She, Robust superhydrophobic attapulgite coated polyurethane sponge for efficient immiscible oil/water mixture and emulsion separation, J. Mater. Chem. A, 2016, 4, 15546-15553.

34 J. Zhang, K. Ji, J. Chen, Y. Ding and Z. Dai, A threedimensional porous metal foam with selective-wettability for oil-water separation, J. Mater. Sci., 2015, 50, 5371-5377.

35 Y. Zhang, Y. Chen, L. Shi, J. Li and Z. Guo, Recent progress of double-structural and functional materials with special wettability, J. Mater. Chem., 2011, 22, 799-815.

36 J. Xu, J. Xu, Y. Cao, X. Ji and Y. Yan, Fabrication of nonflaking, superhydrophobic surfaces using a one-step solution-immersion process on copper foams, Appl. Surf. Sci., 2013, 286, 220-227.

37 Y. L. Zhang, Q. D. Chen, Z. Jin, E. Kim and H. B. Sun, Biomimetic graphene films and their properties, Nanoscale, 2012, 4, 4858-4869.

38 W. Zhang, N. Liu, Y. Cao, X. Lin, Y. Liu and L. Feng, Superwetting porous materials for wastewater treatment: from immiscible oil/water mixture to emulsion separation, Adv. Mater. Interfaces, 2017, 4, 10294-10303.

39 M. Liu, S. Wang, Z. Wei, Y. Song and L. Jiang, Superoleophobic Surfaces: Bioinspired Design of a Superoleophobic and Low Adhesive Water/Solid Interface, Adv. Mater., 2009, 21, 665-669.

40 S. Yang, L. Chen, C. Wang, M. Rana and P. C. Ma, Surface roughness induced superhydrophobicity of graphene foam for oil-water separation, J. Colloid Interface Sci., 2017, 508, 254-262.

41 A. Yurt, S. Ulutas and H. Dal, Electrochemical and theoretical investigation on the corrosion of aluminium in acidic solution containing some Schiff bases, Appl. Surf. Sci., 2006, 253, 919-925. 